HEALTHCARE DELIVERY

\title{
Implementation of electronic scripts in South Africa
}

\author{
K du Toit, S Naicker, J Bodenstein \\ Karen $d u$ Toit is a pharmacist with a PhD in applied chemistry. She is also an Honorary Associate Professor in the Discipline of Pharmaceutical \\ Sciences, University of KwaZulu-Natal, Durban, South Africa (SA), and an admitted attorney. Sarisha Naicker is a pharmacist based in Pretoria \\ with many years' experience in the retail sector. She has been involved in the implementation of electronic scripts in SA. Johannes Bodenstein is a \\ pharmacist with a PhD in pharmacology. He is a senior lecturer in the Discipline of Pharmaceutical Sciences, University of KwaZulu-Natal.
}

Corresponding author: J Bodenstein (bodensteinj@ukzn.ac.za)

The legal framework in South Africa (SA) provides for strict requirements regarding prescriptions. However, pharmacists are still confronted daily with illegible handwritten scripts, increasing the risk of medication errors. E-prescribing is being implemented in SA to overcome these disadvantages. The general regulations made in terms of the Medicines and Related Substances Act as well as the Electronic Communications and Transactions Act must be read conjointly for the purposes of understanding the legal framework of electronic prescriptions in SA.

S Afr Med J 2015;105(9):724-725. DOI:10.7196/SAMJnew.7920

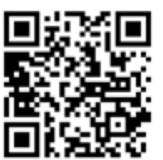

The Oxford Dictionary ${ }^{[1]}$ defines a prescription as a doctor's written instruction authorising a patient to be issued with a medicine or treatment. In South Africa (SA), according to the general regulations made in terms of the Medicines and Related Substances Act, ${ }^{[2]}$ prescriptions must be written in legible print, typewritten or computer generated and signed in person by an authorised prescriber. Despite these requirements, pharmacists are confronted daily with illegible hand-written prescriptions that pose the risk of medication errors. It has been reported in the USA that medication errors resulting from many factors, including illegible handwriting, patient allergies, wrong dosages and drug interactions, account for the deaths of 7000 patients annually, and for nearly 1 in 20 hospital admissions. ${ }^{[3]}$ While no reliable SA data are available, similar safety concerns apply.

In an effort to overcome the disadvantages of written prescriptions, e-prescribing has been implemented in countries such as the USA, with use increasing by $72 \%$ between 2009 and 2010 . Other countries that have adopted e-prescribing are Denmark, Estonia, Iceland, Sweden, Norway, The Netherlands, Greece, England, Scotland, Wales and Northern Ireland. Pilot e-prescribing is currently being undertaken in the Czech Republic, Finland, Italy and Poland. ${ }^{[3]}$ SA has begun the process of implementing e-prescribing. E-prescribing allows a prescriber to electronically send an accurate and understandable prescription directly to a pharmacy from the point of care.

However, the term e-prescribing encompasses more than merely the creation of an electronic script. A complete e-prescribing system requires support software, electronic medication administration records, robots, automated pharmacy systems, bar coding, electronic discharge prescriptions and targeted patient information. ${ }^{[3]} \mathrm{An}$ e-prescribing system serves as an electronic reference handbook. More sophisticated e-prescribing systems act as stand-alone prescription writers. They can create and refill prescriptions for individual patients, manage medications and view patient history, connect to a pharmacy or other drug dispensing site, and integrate with an electronic version of the South African Medicines Formulary $(S A M F)$.

During e-prescribing, a computer-generated prescription is created, validated with an electronic signature, recorded or stored by electronic means, issued and transmitted by electronic means directly from the prescriber to a pharmacist.

\section{The legal framework with regard to electronic scripts}

The Electronic Communications and Transactions Act (ECT Act) ${ }^{[4]}$ came into effect on 30 August 2002. The objectives of this Act are to enable and facilitate electronic communications and transactions in the public interest. The Medicines and Related Substances Act ${ }^{[5]}$ must be read conjointly with the ECT Act for the purposes of understanding the legal framework of electronic prescriptions in SA.

The ECT Act ${ }^{[4]}$ provides that information is not without legal force and effect merely on the grounds that it is wholly or partly in the form of a data message (data message is further defined as data generated, sent, received or stored by electronic means). A computergenerated prescription could therefore have the same legal force as any valid prescription. The requirement that a prescription must be in writing is met if it is in the form of a data message and accessible in a manner usable for subsequent reference. An electronic signature accompanying the script is also not without legal force and effect merely on the grounds that it is in electronic form. An advanced electronic signature is regarded as being a valid electronic signature when applied properly, unless the contrary is proved.

In SA, a faxed, e-mailed, telephonic or electronic prescription must be followed by the original prescription or order within 7 working days. ${ }^{[2]}$ It is questionable whether this requirement will be applicable to e-prescribing, since such scripts will have the same legal force as a written script. In respect of a data message, the addressee is the person who is intended by the originator to receive the data message, but not a person acting as an intermediary in respect of that data message. It is therefore important that an authorised prescriber, with the consent of the patient, sends a data message in the form of a prescription to a chosen pharmacy. Patients have the right to choose their own pharmacy, and any action to the contrary would constitute unprofessional conduct.

Adequate procedures necessary for receipt of an electronic prescription and confirmation of the integrity of communication must be in place. Pharmacists are responsible for verifying the authenticity 
of an electronic prescription, and must be aware of the probable methods of prescription forgery and exercise reasonable care to ensure that prescriptions are genuine. ${ }^{[6]}$ This can be done in a number of ways, such as software programs that require a password, personal identification numbers (PINs), or other methods of authentication. Some programs are able to notify the pharmacist if the message has been tampered with or altered. The pharmacist must assess the integrity of an electronic prescription by judging whether the information remains complete and unaltered, except for the addition of any endorsement and any change arising in the normal course of communication and storage. ${ }^{[5]}$ In the event of the patient keeping the 'original' prescription, or requesting to use a different pharmacy, the e-script can be validated by a barcode on the e-script that is detected by all the different dispensing software programs to enable tracking of the history of the prescription.

In SA, a permanent copy of the faxed, e-mailed, telephonic or other electronic transmitted prescription or order must be made for record purposes and filed. ${ }^{[5]}$ The prescription must be endorsed with necessary changes, such as repeats, confirmation of the prescriber, reasons for not dispensing certain medications, and the request to dispense a generic equivalent. Prescriptions should be retained for 5 years. ${ }^{[2]}$ An electronic script will have to be dealt with in the same manner.

Patient health information must always be collected, recorded and used in a manner that protects confidentiality and privacy. ${ }^{[6]}$ Prescribers and pharmacists must therefore have a secure system for electronic transmission, and equipment used must be placed in secure locations to ensure security and confidentiality of the transmission. Transmissions must also not be delegated to third parties. It must be noted that e-mailed prescriptions from a prescriber to a pharmacy do not ensure the confidentiality of patient records and are not advisable without patient consent.

\section{The advantages and disadvantages of electronic scripts}

Electronic scripts improve patient safety and the quality of care by reducing scripting errors and time spent on phone calls and call-backs to pharmacies. ${ }^{[7]}$ The e-prescribing system ensures the provision of enough specific and required information to fill a prescription. Warning and alert systems at the point of care may assist in averting adverse drug events. The system also improves formulary adherence to the patient's health plan or insurance, permitting substitution of less expensive drug alternatives. ${ }^{[7]}$ Furthermore, automated prescription renewals may be submitted, increasing patient convenience. Clinicians using e-prescribing systems can manage their patients' medications safely and efficiently and also have the convenience of greater prescribing mobility. ${ }^{[7]}$ Furthermore, downloading scripts electronically will not only reduce the patient's waiting time at the pharmacy but allow pharmacists to dispense more scripts per day and to provide an increased professional service and counselling of patients.

Disadvantages of electronic scripts include high costs associated with purchasing, implementing, supporting and maintaining the e-prescribing system, as well as training of staff. System downtime may cause frustration. ${ }^{[7]}$ Security and privacy of patient information may be infringed through errors that lead to accidental disclosure of health information on the internet. Furthermore, new technology makes it easier to alter and forge prescriptions. In SA, challenges such as ensuring that electronic scripts are available in our 11 languages are experienced. Maintenance of correct pack sizes and doses and the elimination of typing errors may also be challenging. ICD-10 codes form part of electronic scripts, and patients may not be comfortable with disclosing their illnesses. The storage of automated repeats often needs to be completed manually. Training of all staff, including locum personnel, may be costly but needs to be achieved. ${ }^{[7]}$

\section{Conclusion}

Electronic scripts significantly enhance safety, are convenient for the prescriber, and save time at the pharmacy. Although the implementation process is potentially troublesome, the advantages of electronic scripts outweigh the disadvantages. E-prescribing would most certainly be more convenient for a patient. Automated prescription renewals can never replace the need for direct 6-monthly evaluation and reassessment of chronic medical conditions by a medical practitioner.

\footnotetext{
1. Waite M, ed. Oxford Dictionary \& Thesaurus. 2nd ed. Oxford: Oxford University Press, 2007.

2. General Regulations made in terms of the Medicines and Related Substances Act No. 101 of 1965 , http://www.saflii.org/za/legis/consol_reg/marsa10101965rangnr510723.pdf (accessed 6 November 2014).

Digitome e-Prescribing. 2011. http://digito.me/eprescribing (accessed 6 November 2014).

4. Electronic Communications and Transactions Act, Act No. 25 of 2002 . http://www.internet.org.za/ 4. Electronic Communications and Transactions
ect_act.html (accessed 6 November 2014).

ect_act.html (accessed 6 November 2014).
5. The Medicines and Related Substances Act 101 of 1965. http://www.hpcsa.co.za/Uploads/editor/ The Medicines and Related Substances Act 101 of 1965. http://www.hpcsa.co.za/Uploads/editor/
UserFiles/downloads/legislations/acts/medicines_and_related_sub_act_101_of_1965.pdf (accessed 19 June 2015).

6. South African Pharmacy Council. Good Pharmacy Practice in South Africa. 4th ed. Pretoria: SAPC, 2010.

7. Cohen J, Banchilhon J-M, Jones M. South African physicians' acceptance of e-prescribing technology: An empirical test of a modified UTAUT model. South African Computer Journal 2013;50:43-54.
}

\section{This month in the $S A M J$}

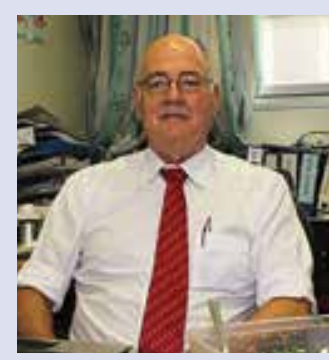

Guy Richards* ${ }^{*}$ is a chief physician in the Department of Medicine and Pulmonology at the University of the Witwatersrand, Johannesburg. He is Academic Head and Professor of the Division of Critical Care and Director of the Department of Critical Care at Charlotte Maxeke Johannesburg Academic Hospital, and currently Chairman of the South African National Critical Care and Thoracic Society Congress. Prof. Richards has been received awards for best research paper, best presentation and best publication on a number of occasions, and has authored 11 book chapters and 126 peer-reviewed scientific papers.

*Richards GA. Nososcomial transmission of viral haemorrhagic fever in South Africa. S Afr Med J 2015;105(9):709-712. [http://dx.doi.org/10.7196/SAMJnew.8168] Richards GA, Weyer J, Blumberg LH. Viral haemorrhagic fevers in South Africa. S Afr Med J 2015;105(9):748-751. [http://dx.doi.org/10.7196/SAMJnew.8330] 